Dielectrophoretic Forces on the Nanoscale

C.M. Schaldach, W.L. Bourcier, P.H. Paul, W.D. Wilson

April 20, 2004 
This document was prepared as an account of work sponsored by an agency of the United States Government. Neither the United States Government nor the University of California nor any of their employees, makes any warranty, express or implied, or assumes any legal liability or responsibility for the accuracy, completeness, or usefulness of any information, apparatus, product, or process disclosed, or represents that its use would not infringe privately owned rights. Reference herein to any specific commercial product, process, or service by trade name, trademark, manufacturer, or otherwise, does not necessarily constitute or imply its endorsement, recommendation, or favoring by the United States Government or the University of California. The views and opinions of authors expressed herein do not necessarily state or reflect those of the United States Government or the University of California, and shall not be used for advertising or product endorsement purposes.

This work was performed under the auspices of the U.S. Department of Energy by University of California, Lawrence Livermore National Laboratory under Contract W-7405-Eng-48. 


\title{
Dielectrophoretic Forces on the Nanoscale
}

\author{
C. M. Schaldach ${ }^{1}$, William L. Bourcier ${ }^{1}$, Phillip H. Paul ${ }^{2}$ and W. D. Wilson ${ }^{1 \#}$ \\ ${ }^{1}$ Lawrence Livermore National Laboratory \\ Livermore, CA 94550 \\ ${ }^{2}$ Eksigent Technologies \\ Livermore, CA 94550 \\ \# To whom correspondence should be sent: \\ Lawrence Livermore National Laboratory \\ 7000 East Avenue, L-232
}

Livermore, CA 94550

(925) 424-2497

wdwils@1lnl.gov 


\begin{abstract}
We have developed a method of calculation of the dielectrophoretic force on a nanoparticle in a fluid environment where variations in the electric field and electric field gradients are on the same nanoscale as the particle. The Boundary Element

Dielectrophoretic Force (BEDF) method involves constructing a solvent-accessible or molecular surface surrounding the particle, calculating the normal component of the electric field at the surface boundary elements and then solving a system of linear equations for the induced surface polarization charge on each element. Different surface elements of the molecule may experience quite different polarizing electric fields, unlike the situation in the point dipole approximation. A single $100 \AA$ radius ring test configuration is employed to facilitate comparison with the well-known point dipole approximation (PDA). We find remarkable agreement between the forces calculated by the BEDF and PDA methods for a $1 \AA$ polarizable sphere. However, for larger particles, the differences between the methods become qualitative as well as quantitative; the character of the force changes from attractive at the origin of the ring for a $50 \AA$ sphere, to repulsive for a $75 \AA$ sphere. Equally dramatic differences are found in a more complex electrical environment involving two sets of 10 rings.
\end{abstract}

Keywords: Dielectrophoresis, Boundary Element, Nanotechnology, membranes, PoissonBoltzmann, electrostatic potentials

\title{
I. Introduction
}

Dielectrophoresis is increasingly being employed to manipulate and separate molecules and particles including biological cells (1-7). Recently, Bennett, et al (1) have driven 
dielectrophoretic phase separations of polystyrene beads and bacteria in microfluidic channels because of induced dipole-dipole interactions. Voldman, et al (2) have investigated how dielectrophoresis-based quadrupole traps for single beads can hold against destabilizing fluid flows. Gascoyne, et al $(3,4)$ have separated cancer cells in blood using dielectrophoretic-driven levitation. Based on the same physics, optical tweezers have been applied to microspheres $(5)$ and cells $(6,7)$, and have even formed the basis of a Nobel Prize (8).

Furthermore, recent developments in nanotechnology enable structures to be built which can create fields and field gradients on unprecedented length scales; the scale of the variations in the field inducing charge on a molecule may be the same as the scale of the molecule itself. Synthetic nanopores have been fabricated in inorganic materials for transporting DNA $(9,10)$. Carbon nanotubes have been aligned in a polymer film to demonstrate molecular transport through their cores $(11,12)$. Dielectrophoresis has recently been employed to assemble nanowires in suspensions (13). Daiguji, et al (14), for example, have studied ion current flow through $30 \mathrm{~nm}$ silica channels which were gated at their midpoint. Using electroless deposition of Au in track-etched pores in polycarbonate films, Martin, et al (15) have constructed membrane channels of the order of molecular dimensions in studies of permselectivity of membranes. Hybrid threedimensional fluidic architectures have been designed which control nanofluidic transfer via surface charged nanopores separating crossed microfluidic channels (16). Perhaps most notably, multilayer technology enables materials comprised of virtually any elements to be constructed with control on atomic dimensions $(17,18)$. 
In the light of these developments in nanoengineering, it seems appropriate to investigate the fundamental assumptions underlying the analytical formulation of dielectrophoretic forces. We have previously presented a method for calculating molecular adsorption $\left(\mathrm{H}_{2} \mathrm{~S}\right.$ and $\mathrm{HS}^{-}$on $\mathrm{Cu}$ ) utilizing boundary elements in a Gouy-Chapman field (19). The method was shown to agree well with the analytic result for a dielectric sphere in a fluid environment (20). Here we extend and apply the method to the calculation of dielectrophoretic forces in situations where the scale of the variations in the field inducing charge on a molecule may be the same as the scale of the molecule, and whose molecular shape may not be spherical. The results are compared to the analytic (pointdipole approximation, PDA) expressions for the dielectrophoretic force (21-23). Sancho, et al (24) and Gheorghiu, et al (25) have recently developed similar methods involving boundary elements for studying shelled particles and cells.

In Section II, we present our method of calculation. Section III contains a comparison between our method and analytic expressions for the dielectrophoretic force when the electric field is due to a simply charged ring. Multiple-ring configurations are compared in Section IV. Section V contains a Summary and Conclusions.

\section{Method of Calculation}

The dielectrophoretic force, $\mathbf{F}$, on a dipole, $\mathbf{p}$, in a non-uniform field, $\mathbf{E}$, is given by (2123)

$$
\mathbf{F}=(\mathbf{p} \bullet \nabla) \mathbf{E}
$$


For a sphere of radius $\mathrm{R}$ having internal dielectric constant $\varepsilon_{2}$ in a dielectric medium of dielectric constant $\varepsilon_{1}$, the effective dipole moment is

$$
\mathbf{p}=2 \pi \mathrm{R}^{3} \boldsymbol{\varepsilon}_{1}\left(\frac{\boldsymbol{\varepsilon}_{2}-\boldsymbol{\varepsilon}_{1}}{\boldsymbol{\varepsilon}_{2}+2 \boldsymbol{\varepsilon}_{1}}\right) \mathbf{E}
$$

where $\frac{\boldsymbol{\varepsilon}_{2}-\boldsymbol{\varepsilon}_{1}}{\boldsymbol{\varepsilon}_{2}+2 \boldsymbol{\varepsilon}_{1}}$ is the well-known Clausius-Mossotti factor for a sphere (20). The dielectrophoretic force in the point-dipole approximation (PDA) becomes

$$
\mathbf{F}=2 \pi \mathrm{R}^{3} \boldsymbol{\varepsilon}_{1}\left(\frac{\boldsymbol{\varepsilon}_{2}-\boldsymbol{\varepsilon}_{1}}{\boldsymbol{\varepsilon}_{2}+2 \boldsymbol{\varepsilon}_{1}}\right) \nabla|\mathbf{E}|^{2}
$$

As Pohl has pointed out (21), Eq. 3 is applicable to a "small" sphere (although not a point dipole, since it has finite radius, R) in a field, $\mathbf{E}$, which field is assumed to be nonuniform enough to produce appreciably different charges on the positive and negative regions of the induced surface polarization charges, but which nevertheless does not vary so strongly as to alter the size of the dipole throughout the sphere. The dielectrophoretic force calculated using Eq. 3 does assume the molecule ("molecule" and "particle" are equivalent for our purposes here) to be a dipole: the magnitudes of the positive and negative induced polarization charges are equal. Eq. 3 is recognized as being applicable to the force on a molecule when the dimensions of the variations in the electrostatic potentials and fields are small compared to molecular dimensions.

An alternative expression for the force on a molecule is (23)

$$
\mathbf{F}=\int \boldsymbol{\sigma}(s) \mathbf{E}(s) d s
$$


where $\sigma(s)$ is the induced surface charge density on an element, s, of the molecular surface and $\mathbf{E}(\mathrm{s})$ is the electric field at the surface element having elemental area ds. Eq. 4 a) is applicable to arbitrary molecular geometry, b) allows for unequal magnitudes of positive and negative charge to be induced (unlike the dipole approximation), and c) takes account of the precise electric field and, hence, field gradient at every element of the molecule. A molecule whose surface elements experience electric fields whose gradients are not representable by the gradient at say, the centroid, may not be able to be represented by the dipole approximation. Given the charges induced on the surface elements, the energy, $\mathrm{W}$, required to bring the molecule from infinity (a position where the field or field gradient is zero) to its position in the nanostructure is given by

$$
W=\frac{1}{2} \int \phi(s) \sigma(s) d s
$$

where $\phi(s)$ is the electrostatic potential at the molecular surface element s.

Our method of calculation, the Boundary Element Dielectrophoretic Force (BEDF) method, involves first constructing a molecular or solvent accessible surface surrounding the molecule by a method we have previously described (19). This surface provides the interface between the dielectric media and the molecule; elements of the surface are assigned a unit normal and an elemental area. The electric field, E, created by the nanostructure provides a source of polarization of the molecule. The induced interfacial charge, $\sigma$, can be obtained from a straightforward consideration of the electrostatic boundary conditions and self -terms. This leads to a system of linear equations $(26,19)$,

$$
\{[\mathbf{I}]-f[\mathbf{K}]\}[\boldsymbol{\sigma}]=f[\mathbf{E} \bullet \mathbf{n}]
$$


where

$$
K_{i k}=\int \frac{\mathbf{r}_{i k} \bullet \mathbf{n}_{i}}{\left|\mathbf{r}_{i k}\right|^{3}} d S_{k}
$$

$\mathbf{r}_{i k}$ is the vector distance between elements $\mathrm{i}$ and $\mathrm{k}$ on the molecular boundary; $\mathbf{n}_{i}$ is the outward normal at boundary element $\mathrm{i} ; d S_{k}$ is the differential associated with the area of boundary element $\mathrm{k} ; \mathbf{E} \bullet \mathbf{n}$ is the column vector of normal components of the electric field. $\mathrm{f}$ is given by,

$$
f=\frac{\varepsilon_{2}-\varepsilon_{1}}{2 \pi\left(\varepsilon_{1}+\varepsilon_{2}\right)}
$$

( $\varepsilon_{2}=1.0$ here, but is not restricted to this) and $\varepsilon_{1}$ is the dielectric constant of the solvent ( $\varepsilon_{1}=78.5$ for water here, but, again, is not restricted to this.).

Solution of Eq. [6] by the usual methods of linear algebra provides the polarization charge, $\sigma$, created by $\mathbf{E}$ at each surface element. In this way, different surface elements of the molecule are allowed to experience quite different polarizing electric fields, unlike the situation in the point dipole approximation. The field gradient, variations in the field over the scale of the molecule, is properly taken into account.

\section{Molecule in the vicinity of a ring}

A simple charged ring of radius $\mathrm{R}=100 \AA$ provides an interesting test case. The axial electric field has variations over length scales of the order of its radius which serve to illustrate several important features. The potentials and fields can be calculated 
analytically (20) and can also be obtained numerically by constructing the ring of small Debye-Huckel (27) atoms (spheres) and performing a direct summation of their individual contributions (28). We employed the numerical method here to facilitate our investigation into more complicated structures to be described below. In these demonstration calculations, each atomic element of the ring was given a charge of 0.1 electrons; the magnitude of the fields and forces in this test case are small but obviously scale with the charge.

In Fig. 1, we have plotted the magnitude of the electric field, $|\mathbf{E}|$, due to the charged ring of radius $\mathrm{R}=100 \AA$ (the ring is in the $\mathrm{x}-\mathrm{y}$ plane, centered at $(\mathrm{x}, \mathrm{y}, \mathrm{z})=(0,0,0))$ as a function of the distance, $\mathrm{z}, \mathrm{a}$ ) along its axis, $\mathrm{x}=\mathrm{y}=0.0$ (solid line, filled circles) and also $\mathrm{b}$ ) along an axis at $\mathrm{x}=75.0 \AA, \mathrm{y}=0$ (solid line alone). It is interesting to note that $|\mathbf{E}|$ along the $\mathrm{x}=0$ axis has peaks, maxima, at $\mathrm{z}_{\mathrm{m}}= \pm(\operatorname{sqrt}(2) / 2) \mathrm{R}(\sim 70.7 \AA)$ due to the symmetry-induced dominance of the $\mathrm{z}$-component of the field. On the other hand, along the $\mathrm{x}=75.0 \AA$ axis, a single maximum in $|\mathbf{E}|$ is seen at $\mathrm{z}=0.0$. This shift in the peak of the magnitude of the field is caused by the increase in the off-axis $x$-component at $x=75.0 \AA$, also plotted in Fig. 1 (short-dashed line). Note that the $x$-component also exhibits small peaks at $z=84.1$ $\AA$ (150 Bohr). (The y-component of the electric field is zero in both cases because of the symmetry.) The $\mathrm{z}$-component of the electric field along the $\mathrm{x}=75.0 \AA$ axis, also shown in Fig. 1 (long-dashed line), changes sign at $\mathrm{z}=0$ and has its own extrema at $\pm 25.4 \AA( \pm 48$ Bohr); nevertheless, the $\mathrm{x}$-component, peaked at $\mathrm{z}=0.0$, dominates the field magnitude off-axis ( $\mathrm{x}=75.0 \AA)$. 
Next, the dielectrophoretic force (DF) on a sphere of radius $\mathrm{R}_{\mathrm{m}}=1 \AA$ was calculated as a function of its axial distance, $\mathrm{z}$, along the ring axis $(\mathrm{x}=\mathrm{y}=0.0)$ using both the point-dipole approximation (PDA, Eq. 3 above) and the BEDF method (Eq. 4 above). The results of this comparison are shown in Fig. 2. It is first of all to be noted that, for this small molecule, these very different methods of calculation are in remarkable agreement with each other. The DF is negative for small $\mathrm{z}>0$ (molecule is being attracted backward toward the ring center) and positive for small $\mathrm{z}<0$ (molecule is being attracted toward the ring center) indicating that the molecule is trapped at the ring center. As we will discuss in greater detail later, in the region $-\mathrm{z}_{\mathrm{m}}<\mathrm{z}<\mathrm{z}_{\mathrm{m}}$, the induced polarization charge on the surface elements of the sphere nearest the ring center is found by direct solution to Eq. 6 to be positive, while those elements furthest from the ring are determined to be negatively charged. The electric field in this region is growing in magnitude (see Fig. 1) and, although there is an excess of positive charge induced (more about this below) the force on the negative charge exceeds that on the positive charge, making the total force negative as indicated in Fig. 2. When the molecule is along the $\mathrm{z}$-axis at $\mathrm{z}>\mathrm{z}_{\mathrm{m}}$, the sign of the induced polarization charges is as for $\mathrm{z}<\mathrm{z}_{\mathrm{m}}$ but now the magnitude of the electric field is diminishing. Consequently, the positive surface charge interacts with a larger electric field and the total force on the molecule becomes positive. Note from Fig. 2 that this occurs at $\mathrm{z}=\mathrm{z}_{\mathrm{m}}$ where the peak in the magnitude of the electric field, $|\mathbf{E}|$, is seen to occur (see Fig. 1). Similar effects occur at $\mathrm{z}<-\mathrm{z}_{\mathrm{m}}$. Whereas the region near the ring origin is attractive, the region near the extrema in the electric field is repulsive. The dielectrophoretic energy calculated from the induced charges and the electrostatic 
potential (Eq. 5 above), also plotted in Fig. 2 (solid line, open circles) reflects these effects: For example, a minimum in the energy is seen to occur where the force has a negative slope as it goes through zero, but an energy maximum occurs when the force goes through zero with positive slope.

Note, from Fig. 2, there are deviations between the point-dipole and BEDF methods near the extrema. The point -dipole approximation gives consistently larger magnitude forces than the BEDF method. This deviation is caused by the dipole (equal positive and negative charges) approximation. We find that for $-\mathrm{z}_{\mathrm{m}}<\mathrm{z}<\mathrm{z}_{\mathrm{m}}$ there is an excess of positive charge induced (resulting from the $\mathrm{x}$-component of the field) on the elements nearest the origin while for $|z|>z_{m}$ an excess of negative charge is induced on those elements furthest from the origin. These excess charges in each case interact with the lower magnitude electric field at those elements, thus leading to a slightly reduced force. This excess charge effect becomes more serious for larger molecules (particles), as we will see.

In Fig. 3a, we have plotted the dielectrophoretic force on spheres of radius $50 \AA$ and $75 \AA$ as a function of distance, $\mathrm{z}$, along the ring axis $(\mathrm{x}=\mathrm{y}=0)$, again calculated in the pointdipole approximation (PDA) and by the BEDF method. The two methods give similar characteristics, albeit different magnitudes, for the $50 \AA$ sphere: Positive force for small z $<0$ and negative force for small $\mathrm{z}>0$ as found for the $1 \AA$ sphere in Fig. 2. As for the 1 $\AA$ sphere, the point-dipole approximation predicts higher magnitude forces because of the $\mathrm{x}$-component of the off-axis electric field at the molecular surface elements. At $\mathrm{x}=75 \AA$, the surface elements of the sphere experience dramatically different electric fields (even a 
shift in the peaks of $|\mathbf{E}|$ : see Fig. 1) which dramatically change the character of the dielectrophoretic force. We see in Fig. 3a, that the slope of the force vs distance curve changes sign. This is a quantitative and qualitative change in the force from

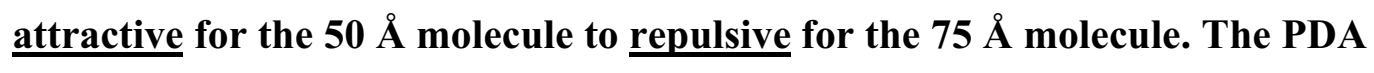
predicts both molecules to be attracted toward the center. In addition, the PDA predicts repulsion at the peaks in the field, i.e., the slope of the DF vs distance curve is positive as the force goes through zero, while the BEDF method exhibits no such repulsive behavior at the peaks.

In Fig. 3b we expand the inset region noted in Fig. 3a for $50 \AA$ and $75 \AA$ spheres and include the dielectrophoretic force for a $65 \AA$ sphere. This intermediate-sized sphere shows a weak attraction, reduced by an order of magnitude from the force on the $50 \AA$ sphere and shows the transition in the slope of the force taking place as a function of sphere radius. It is interesting to note from Fig. $3 \mathrm{~b}$ that the dielectrophoretic force on a 50 $\AA$ sphere moved along $\mathrm{z}$ at the $\mathrm{x}=30 \AA$ off-axis position exhibits similar behavior to the $65 \AA$ sphere moved on-axis. As the off-center sphere moves far from the ring center (not shown), it follows the behavior for the $50 \AA$ on-axis sphere.

As we have alluded to above, a better understanding of these effects involves the induced surface polarization charges (more precisely, the normal component of the electric field at molecular surface elements, see Eq. 6.) In Fig. 4, we have plotted the positive and negative surface polarization charges on the elements of the $1 \AA$ and $75 \AA$ spheres as a function of the position of the centroid of each molecule. (These charges are obviously separated from each other.) For the $1 \AA$ sphere, we find two extrema each for the positive and negative charges, these extrema coinciding with the maxima in the magnitude of the 
electric field, $\mathrm{z}_{\mathrm{m}}$, for the $1 \AA$ sphere shown in Fig. 1 . For the $75 \AA$ sphere, the positive and negative induced charges have a markedly different character: a single extremum at the ring origin $(\mathrm{z}=0)$ is found for each.

In Fig. 5, we sum these positive and negative charges and plot the total induced charges on each of the two spheres as a function of the position of their centroids along the ring axis. For both spheres, we find an increase in total induced positive charge near the origin (lines with open and closed circles) resulting from the x-component of the electric field (actually, from the large normal component, $\mathbf{E} \bullet \mathbf{n}$, see Eq. 6 and Fig. 1). As discussed above, for the small sphere, the negative charge induced on the leading edge of the molecule as it moves toward positive $\mathrm{z}$ values interacts with the large electric field at those leading elements resulting in a negative force. The excess positive charge induced by the $\mathrm{x}$-component of the field on the small sphere multiplied by the z-component of the field at those elements is insufficient to overcome this negative force. On the other hand, in the case of the $75 \AA$ sphere, there is a large increase in positive charge on elements of the sphere near the origin ( $\mathbf{E} \bullet \mathbf{n}$ again), and now this increase in positive charge, even though multiplied by a smaller field, is sufficient to overcome the larger field at the leading negative elements of the molecule. The character of the force changes from attractive to repulsive.

Additional understanding of this effect comes from performing similar calculations, determining the induced charges, in the absence of an x-component of the field. In Fig. 5, then, we also have plotted the total induced charges for the $1 \AA$ and $75 \AA$ sphere when the field is (fictitiously) entirely along the z-axis (lines with open and closed squares). Here we find an excess negative charge is induced for $-\mathrm{z}_{\mathrm{m}}<\mathrm{z}<\mathrm{z}_{\mathrm{m}}$ consistent with the larger 
field magnitude being near the origin, while for $|z|>z_{m}$ the induced polarization charge is positive, again consistent with the magnitude of the field at those furthermost elements from the origin. The magnitude of this effect is greater for the larger sphere; the point along $\mathrm{z}$ where the sign change occurs also differs somewhat because of the size, i.e., the location of the elements at which the charge is being induced. These calculations provide direct evidence of the influence of the $\mathrm{x}$-component of the field on the induced charges and hence the character of the dielectrophoretic force.

The point-dipole approximation assumes the molecule to be spherical (ClausiusMossotti); the BEDF method presented here allows us to investigate shape effects. To this end, using the method described above, we constructed a molecular surface surrounding a 10 x 10 atom planar molecule ("face-centered-cubic-like" geometry, radius of each atom, $1.2 \AA$ ) and then calculated the dielectrophoretic force on that planar molecule in the same ring environment as above. In Fig. 6, we present the results of these calculations when the plane of the molecule is the same as the ring (labeled "parallel" in Fig. 6) and also when the molecule is rotated 90 degrees about the $\mathrm{x}$-axis so its normal is perpendicular to the plane of the ring ("perpendicular" in Fig. 6). Both orientations give rise to attractions to the origin and repulsions at the maxima of the electric field $\left(z_{\mathrm{m}}\right.$ in Fig. 1), the force changing sign appropriately at these critical points. The parallel orientation results in forces which can be a factor of 2-3 greater than the parallel configuration. From Fig. 6, we find the binding energy (difference in energy between the energy maxima and minima) for the parallel configuration is nearly twice the binding of the perpendicular geometry. These calculations indicate that shape effects must be considered when calculating dielectrophoretic forces on this scale. 


\section{Molecule in a complex ring environment}

As discussed above, nanotechnology has enabled interesting and technologically relevant geometrical configurations to be produced. Notable among these are multilayers or nanolaminates $(17,18)$ where atomic-scale layers of different materials can be produced adjacent to one another with single atom interfaces between them. Optimizing a nanoscale geometry is beyond the scope of this work; we calculated the electric fields for a set of rings which is illustrative of the enabling power of the technology while providing further examples of the need to calculate dielectrophoretic forces using a molecular theory appropriate to these nanoscale configurations.

When multiple rings are employed, the fields become more interesting. $\mathrm{N}$ rings of the same charge placed next to each other result in both an increase in the magnitude of the resulting field and a decrease in the position of the extrema relative to the plane of the end ring. An oppositely charged set of $\mathrm{N}$ rings could be configured along the same axis as the first at a distance chosen to optimize the magnitude of the field in-between the sets of rings.

A single set of 10 coaxial rings, axis along $z$, radius $100 \AA$, comprised of atoms each having a charge of +0.1 electrons as for the single ring above, placed next to each other in a row, will produce the electric field profile shown in Fig. 7. The set, or layer, has a charge density of $\sim 3.4 \mu \mathrm{C} / \mathrm{cm}^{2}$. The "leftmost" ring is placed (in the $\mathrm{x}-\mathrm{y}$ plane) at $\mathrm{z}=0$; the electric field is zero in the middle of the 10-ring configuration (at 19 Bohr $=10.1 \AA$ ) and directed positively along the $\mathrm{z}$-axis. A second 10-ring configuration, this time negatively charged, coaxial with the first (see Fig. 7) will also produce an electric field 
directed positively along the z-axis. By spacing the 10-ring sets so the extrema in the $\mathrm{z}$ component of their electric fields coincide (separation $=123.3 \AA$ (233 Bohr)), we optimize the field at 154 Bohr ( $81.5 \AA$ ), the mid-point between the rings (see Fig. 7). As can be seen in Fig. 7, this $2 \times 10$ ring configuration also gives rise to zeroes in the field at $-15.3 \AA$ ( $-29 \mathrm{Bohr})$ and $177.8 \AA$ (336 Bohr) as well as secondary peaks at $-73.6 \AA$ (-139 Bohr) and $236.0 \AA$ (446 Bohr).

The off-axis electric field for this $2 \times 10$ ring configuration is dramatically different from that along the axis. In Fig. 7, we have also plotted the x-component and magnitude of the electric field for the $2 \times 10$ ring configuration along the $\mathrm{z}$-axis at $\mathrm{x}=75 \AA$. The large $\mathrm{x}-$ component in the middle of the rings is responsible for the double-peaked character of the magnitude.

In Fig. 8, we show the results of our dielectrophoretic force calculations for a $75 \AA$ and $90 \AA$ sphere in the $2 \times 10$ ring environment described above using the BEDF method and, for comparison, the PDA method for the $75 \AA$ sphere. (The PDA method for the $90 \AA$ sphere shows the same behavior as the $75 \AA$, with its magnitude adjusted for the radius.) The PDA predicts an attraction (negative slope of DF vs $\mathrm{z}$ as it goes through zero) where the electric field is zero (-29 Bohr and 336 Bohr) and a repulsion (positive slope of DF vs $\mathrm{z}$ as it goes through zero) where the secondary peaks in $|\mathbf{E}|$ occur (see Fig. 7). The BEDF method predicts no attractions or repulsions in these regions. Strikingly, at the maximum in the $|\mathbf{E}|$ field for the $2 \times 10$ ring, (see $\mathrm{x}=0.0|\mathbf{E}|$ field ( $\mathrm{z}=154$ Bohr) in Fig. 7), the BEDF and PDA methods applied to the $75 \AA$ sphere agree as to the repulsive character of the force (positive slope of DF vs $\mathrm{z}$ as it goes through zero). However, the methods produce opposite characters for the force on a $90 \AA$ sphere: The dielectrophoretic force on a $90 \AA$ 
sphere in the field maximum is attractive according to the BEDF method. The PDA is probing the field and field gradient along the axis of the $2 \times 10$ ring configuration, while the molecular surface elements at which the charge is being induced are probing quite different regions because of the scale of the field variations (see Fig. 7).

\section{Summary and Conclusions}

With the advent of modern engineered nanostructures, it is vital to employ a method of calculation of the dielectrophoretic forces on nearby molecules which is commensurate with the scale of the structure. The point-dipole approximation was never intended to be employed where variations in the electric field occur on the same scale as that of the molecule or particle (21-23). We find remarkable agreement between the forces calculated by our boundary element dielectrophoretic force method (BEDF) method and those obtained in the point-dipole approximation (PDA) for small molecules. For larger molecules, however, we find quantitative and qualitative differences between the methods. We have presented results for a simple theoretical example in this work, that of a ring, which clearly show the pitfalls in employing the PDA: The dipole approximation itself does not hold, i.e., there can be an excess of charge of one sign induced on the molecule. This can lead to a change in the character of the force in various regions of the ring, attractive vs repulsive, in direct disagreement with the PDA. Furthermore, shape effects are naturally included in the BEDF method and, for a simple example of a planar $10 \times 10$ atom molecule, found to introduce a factor of $\sim 2-3$ difference in the force depending upon orientation of the molecule. 
Beyond the simple ring example, we considered a possible nanoengineered structure motivated by the technology of multilayers, that of two oppositely charged layers of 10 rings each. Here the forces are large enough to be of technological relevance. Again, we find dramatic differences in the character of the forces between our BEDF and the PDA methods. Where attractions and repulsions are found in regions where the electric field changes sign using the PDA, no such forces are obtained for the BEDF.

Because of the subtleties involved in predicting the induced surface charge on elements of a molecule, it is difficult to know beforehand where the point dipole approximation might break down. This necessitates the use of the theory appropriate to the scale of the problem. 


\section{References}

(1) Bennett, D.J.; Khusid, B.; James, C.D.; Galambos, P.C.; Okandan, M.; Jacqmin, D.; Acrivos, A. Appl. Phys. Letts. 2003, 83, 4866.

(2) Voldman, J.; Braff, R.A.; Toner, M.; Gray, M.L.; Schmidt, M.A. Biophys. J. 80 (2001) 531.

(3) Gascoyne, P.R.C.; Vykoukal, J. Electrophoresis 2002, 23, 1973.

(4) Gascoyne, P.R.C.; Wang, X.-B.; Huang, Y.; Becker, F.F. IEEE Trans. Ind. Appl. 1997, 33, 670 .

(5) Wright, W.H.; Sonek, G.J.; Berns, M.W. Appl. Optics 1994, 33, 1735.

(6) Fuhr, G.; Arnold, W.M.; Hagedorn, R.; Muller, T.; Benecke, W.; Wagner, B.;

Zimmermann, U. Biochim. Biophys. Acta. 1992, 1108, 215.

(7) Schnelle, T.; Muller, T.; Hagedorn, R.; Voigt, A.; Fuhr, G. Biochim. Biophys. Acta. 1999, $1428,99$.

(8) Chu, S. "for development of methods to cool and trap atoms with laser light", Nobel

Prize in Physics, 1997; Optical tweezers reviewed in Molloy, J.E.; Padgett, M.J. Contemporary Physics 2002, 43, 241.

(9) Meller, A.; Nivon, L.; Brandin, E.; Golovchenko, J.; Branton, D. Proc. Natl. Acad. Sci. 2001, 97, 1079.

(10) Li, J.; Stein, D.; McMullan, C.; Branton, D.; Aziz, M.J.; Golovchenkok, J. Nature 2001, 412, 166.

(11) Hinds, B.J.; Chopra, N.; Rantell, T.; Andrews, R.; Gavalas, V.; Bachas, L.G. Science $\mathbf{2 0 0 4}, 303,62$.

(12) Noy, A.; Bakajin, O. private communication. 
(13) Hermanson, K.D.; Lumsdon, S.O.; Williams, J.P.; Kaler, E.W.; Velev, O.D. Science 2001, 294, 1082.

(14) Daiguji, H.; Yang, P.; Majumdar, A.; NanoLett. 2004, 4, 137.

(15) Martin, C.R.; Nishizawa, M.; Jirage, K.; Kang, M.; Lee, S.B. Adv. Mater. 2001, 13, 1351.

(16) Kuo, T.-C.; Cannon, Jr., D.M.; Chen, Y.; Tulock, J.J.; Shannon, M.A.; Sweedler, J.V.; Bohn, P.W. Anal. Chem. 2003, 75, 1861.

(17) Bajt, S.; Alameda, J.B.; Barbee, T.W.; Clift, W.M.; Folta, J.A.; Kaufmann, B.; Spiller, E.A. Optical Eng. 2002, 41, 1797.

(18) Spiller, E.; Baker, S.L.; Mirkarimi, P.B.; Sperry, V.; Gullikson, E.M.; Stearns, D.G. Appl. Opt. 2003, 42, 4049.

(19) Wilson, W.D.; Schaldach, C.M. J. Coll. Interface Sci. 1998, $208,546$.

(20) Jackson, J.D. Classical Electrodynamics, 3rd ed.; John Wiley and Sons: New York, 1999.

(21) Pohl, H.A. Dielectrophoresis; Cambridge University Press: Cambridge, 1978.

(22) Jones, T.B. Electromechanics of Particles; Cambridge University Press: Cambridge, 1995.

(23) Jones, T.B. J. Electrostat. 1979, 6, 69.

(24) Sancho, M.; Martinez, G.; Martin, C. J. Electrostat. 2003, 57, 143.

(25) Gheorghiu, E. Ann. N. Y. Acad. Sci. 1999, 873, 262.

(26) Zauhar, R.J.; Morgan, R.S. J. Mol. Biol. 1985, 186, 815. See also J. Comp. Chem. 1990, 11, 603; J. Comp. Chem. 1988, 9, 171; J. Comp. Chem. 1991, 12, 575.

(27) Debye, P.; Hückel, E. Physik Z. 1923, 24, 185. 
(28) Schaldach, C.M.; Bourcier, W.L.; Paul, P.H.; Wilson, W.D. J. Coll. Interface Sci. 2004, manuscript accepted for publication. 


\section{Figure Legends}

Figure 1: Magnitude of the electric field, $|\mathbf{E}|$, due to a charged ring of radius $R=100 \AA$ as a function of the distance, $\mathrm{z}, \mathrm{a}$ ) along its axis, $\mathrm{x}=\mathrm{y}=0.0$ (solid line, filled circles) and also b) along an axis at $\mathrm{x}=75.0 \AA \mathrm{y}=0$ (solid line alone). Note that $|\mathbf{E}|$ along the $\mathrm{x}=0$ axis has maxima at $\mathrm{z}_{\mathrm{m}}= \pm(\operatorname{sqrt}(2) / 2) \mathrm{R}(\sim 70.7 \AA)$; along the $\mathrm{x}=75.0 \AA$ axis, a single maximum in $|\mathbf{E}|$ is seen at $\mathrm{z}=0.0$. This shift in the peak of the magnitude of the field is caused by the increase in the off-axis $\mathrm{x}$-component at $\mathrm{x}=75.0 \AA$ (short-dashed line). The $\mathrm{z}$-component of the electric field along the $\mathrm{x}=75.0 \AA$ axis (long-dashed line), changes sign at $\mathrm{z}=0$ and has its own extrema at $\pm 25.4 \AA$ ( \pm 48 Bohr); nevertheless, the x-component, peaked at $\mathrm{z}=0.0$, dominates the field magnitude off-axis $(\mathrm{x}=75.0 \AA)$.

Figure 2: Dielectrophoretic force (DF) on a sphere of radius $\mathrm{R}_{\mathrm{m}}=1 \AA$ as a function of its axial distance, $\mathrm{z}$, along the ring axis $(\mathrm{x}=\mathrm{y}=0.0)$ using both the point-dipole approximation (PDA, Eq. 3) and the BEDF method (Eq. 4). Note that, for this small molecule, these very different methods of calculation are in remarkable agreement with each other. The dielectrophoretic energy calculated from the induced charges and the electrostatic potential (Eq. 5), (solid line, open circles) exhibits a minimum in the energy where the force has a negative slope as it goes through zero, but an energy maximum occurs when the force goes through zero with positive slope.

Figure 3: Dielectrophoretic force on spheres of radius $50 \AA$ and $75 \AA$ as a function of distance, $\mathrm{z}$, along the ring axis calculated in the point-dipole approximation (PDA) and by the BEDF method. The two methods give similar characteristics, albeit different magnitudes, for the $50 \AA$ sphere. For the $75 \AA$ sphere, however, the slope of the force vs 
distance curve changes sign. This is a quantitative and qualitative change in the force from attractive for the $50 \AA$ molecule to repulsive for the $75 \AA$ molecule. The PDA predicts both molecules to be attracted toward the center. In Fig. $3 \mathrm{~b}$ we expand the inset region noted in Fig. 3a for $50 \AA$ and $75 \AA$ spheres and include the dielectrophoretic force for a $65 \AA$ sphere which shows the transition in the slope of the force taking place as a function of sphere radius.

Figure 4: Positive and negative surface polarization charges on the elements of the $1 \AA$ and $75 \AA$ spheres as a function of the position of the centroid of each molecule. For the $1 \AA$ sphere, we find two extrema each for the positive and negative charges, these extrema coinciding with the maxima in the magnitude of the electric field, $z_{m}$, for the $1 \AA$ sphere shown in Fig. 1. For the $75 \AA$ sphere, the positive and negative induced charges have a markedly different character: a single extremum at the ring origin $(\mathrm{z}=0)$ is found for each.

Figure 5: Total induced charges on each of the $1 \AA$ and $75 \AA$ spheres as a function of the position of their centroids along the ring axis. For both spheres, we find an increase in total induced positive charge near the origin (lines with open and closed circles) resulting from the x-component of the electric field. We also have plotted the total induced charges for the $1 \AA$ and $75 \AA$ sphere when the field is (fictitiously) entirely along the z-axis (lines with open and closed squares). These calculations provide direct evidence of the influence of the x-component of the field on the induced charges and hence the character of the dielectrophoretic force (see text).

Figure 6: Shape effects: Dielectrophoretic force on a planar molecule in the same ring environment as in Fig. 1 when the plane of the molecule is the same as the ring (labeled 
"parallel" in Fig. 6) and also when the molecule is rotated 90 degrees about the x-axis so its normal is perpendicular to the plane of the ring ("perpendicular" in Fig. 6). The parallel orientation results in forces which can be a factor of 2-3 greater than the parallel configuration. The binding energy (difference in energy between the energy maxima and minima) for the parallel configuration is nearly twice the binding of the perpendicular geometry. These calculations indicate that shape effects must be considered when calculating dielectrophoretic forces on this scale.

Figure 7: Electric field profile due to single set of 10 coaxial rings (closed circles), axis along z, radius $100 \AA$, comprised of atoms each having a charge of +0.1 electrons. The "leftmost" ring is placed (in the $x-y$ plane) at $\mathrm{z}=0$; the electric field is zero in the middle of the 10-ring configuration. A second 10-ring configuration, this time negatively charged, coaxial with the first (closed squares) is spaced so the extrema in the zcomponent of their electric fields coincide; we optimize the field at 154 Bohr $(81.5 \AA)$, the mid-point between the rings. The off-axis electric field for this $2 \times 10$ ring configuration is dramatically different from that along the axis (dashed and closed lines). The large $\mathrm{x}$-component in the middle of the rings is responsible for the double-peaked character of the magnitude of the field at $\mathrm{x}=75 \AA$.

Figure 8: Dielectrophoretic force calculations for a $75 \AA$ and $90 \AA$ sphere in a 2 x 10 ring environment (see Fig. 7) using the BEDF method and, for comparison, the PDA method for the $75 \AA$ sphere. Strikingly, at the maximum in the $|\mathbf{E}|$ field for the $2 \times 10$ ring, (see $\mathrm{x}=0.0|\mathbf{E}|$ field $(\mathrm{z}=154$ Bohr $)$ in Fig. 7), the BEDF and PDA methods applied to the $75 \AA$ sphere agree as to the repulsive character of the force. However, the methods produce opposite characters for the force on a $90 \AA$ sphere: The dielectrophoretic force on a $90 \AA$ 
sphere in the field maximum is attractive according to the BEDF method. The PDA is probing the field and field gradient along the axis of the $2 \times 10$ ring configuration, while the molecular surface elements at which the charge is being induced are probing quite different regions because of the scale of the field variations (see Fig. 7). 




Fig. 1

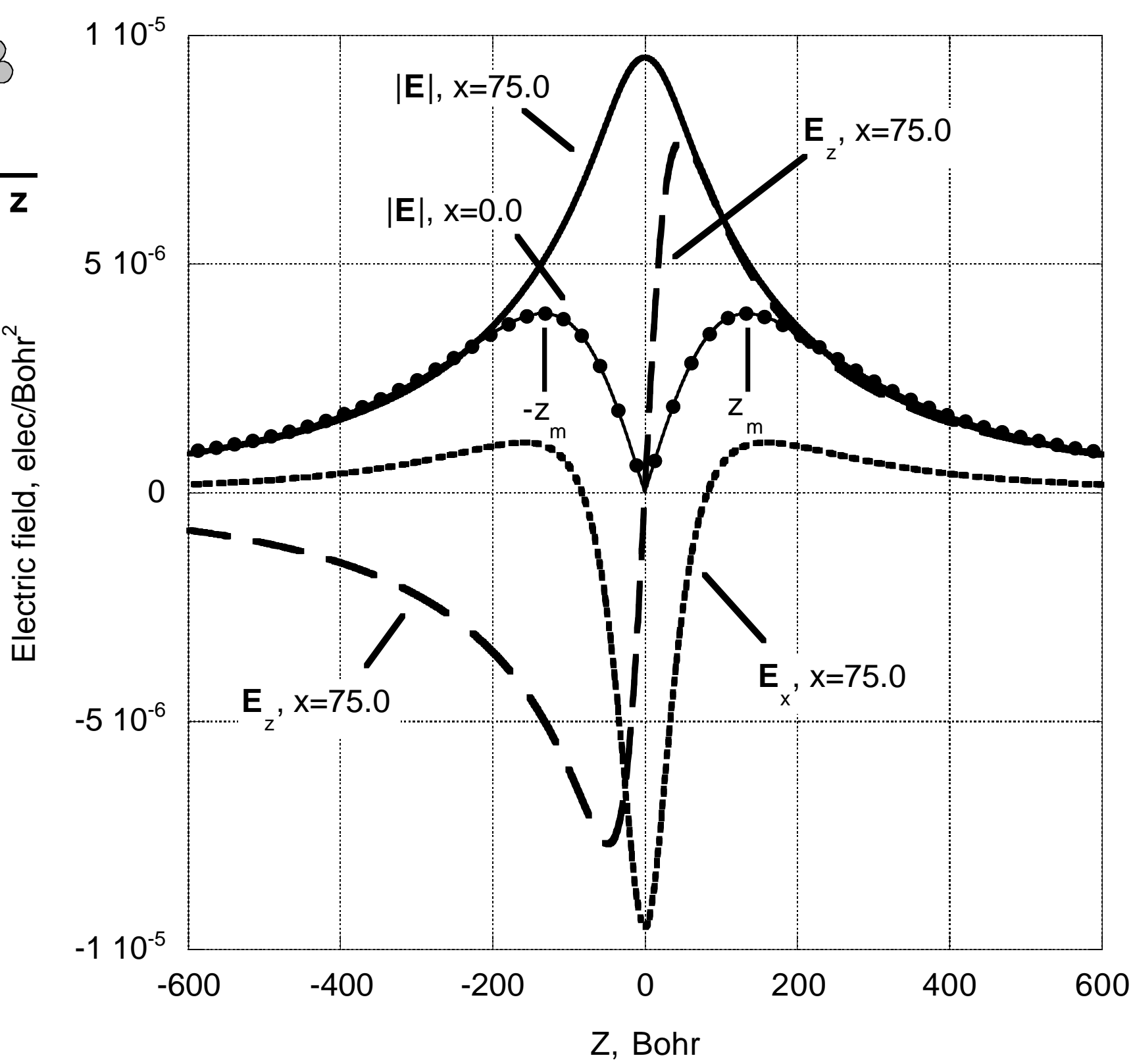


Fig. 2

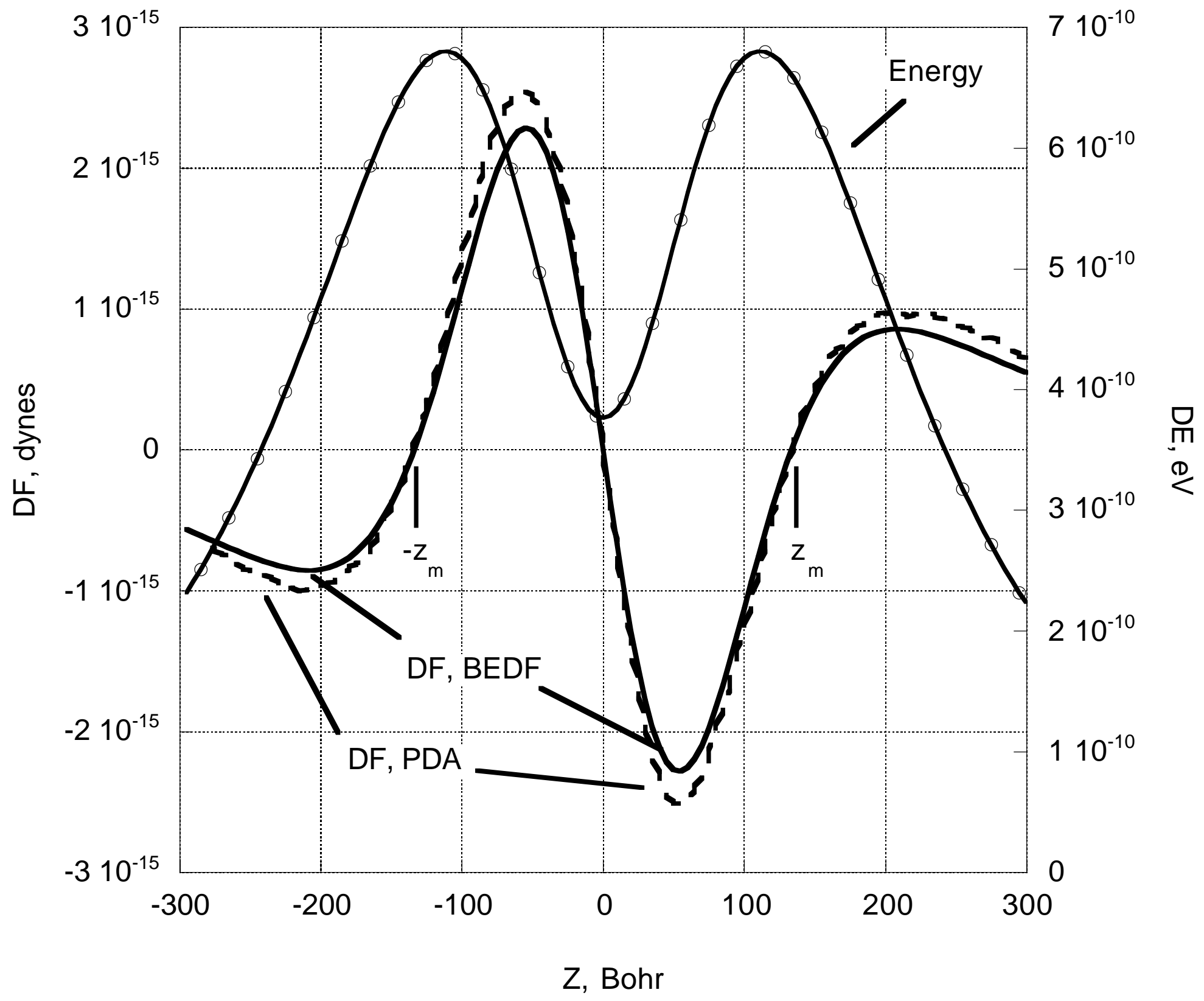


a

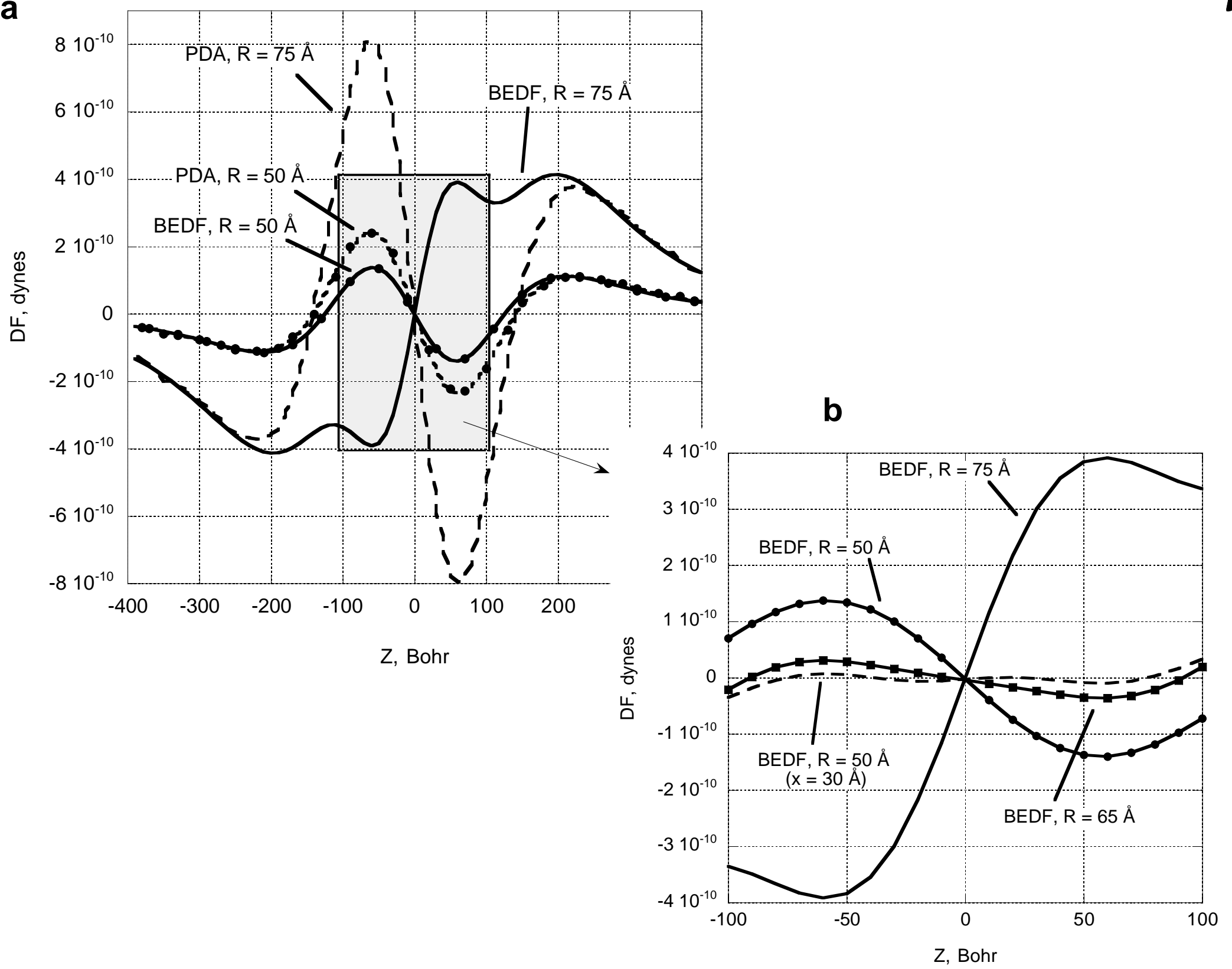

Fig. 3 
Fig. 4

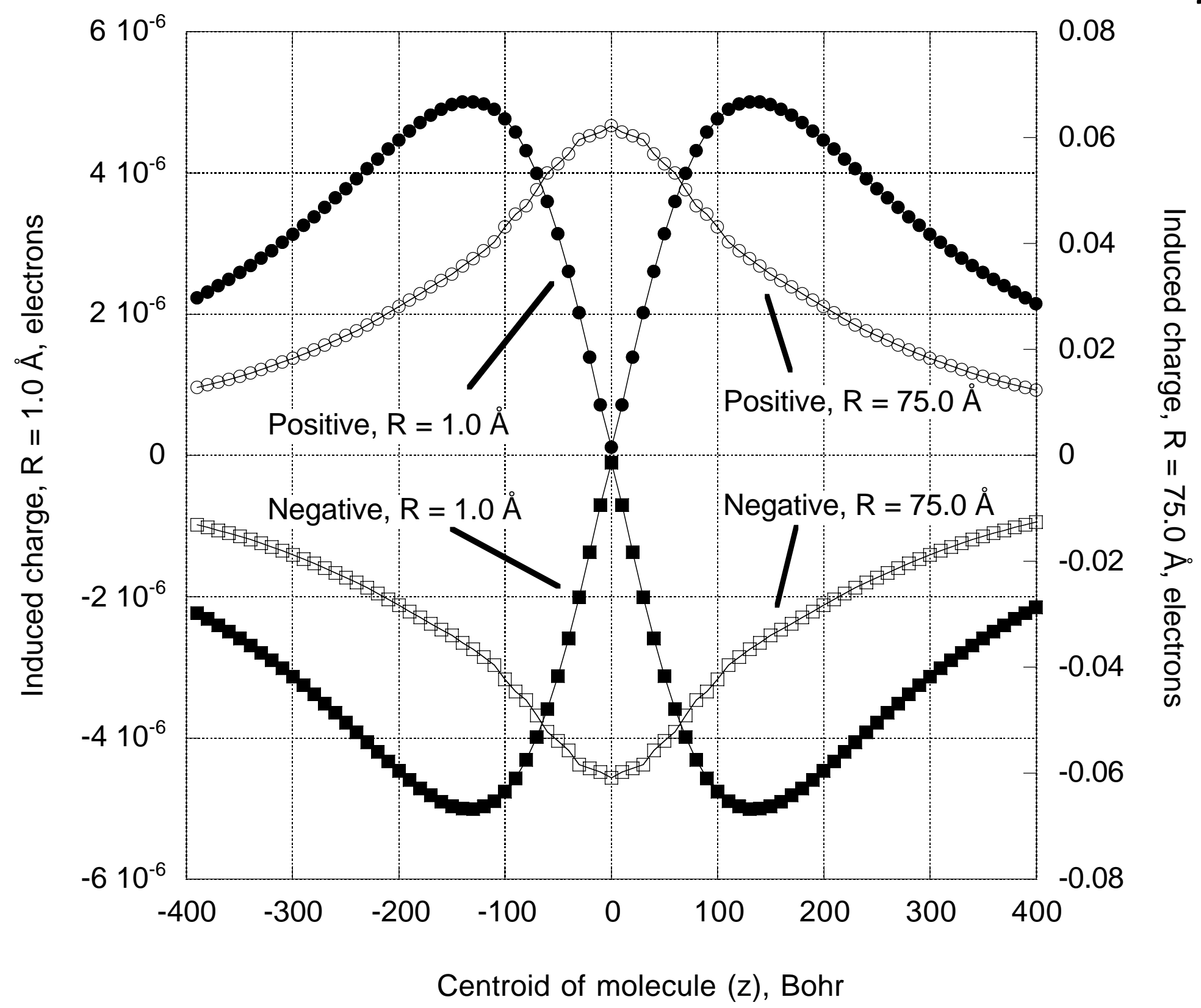


Total charge,

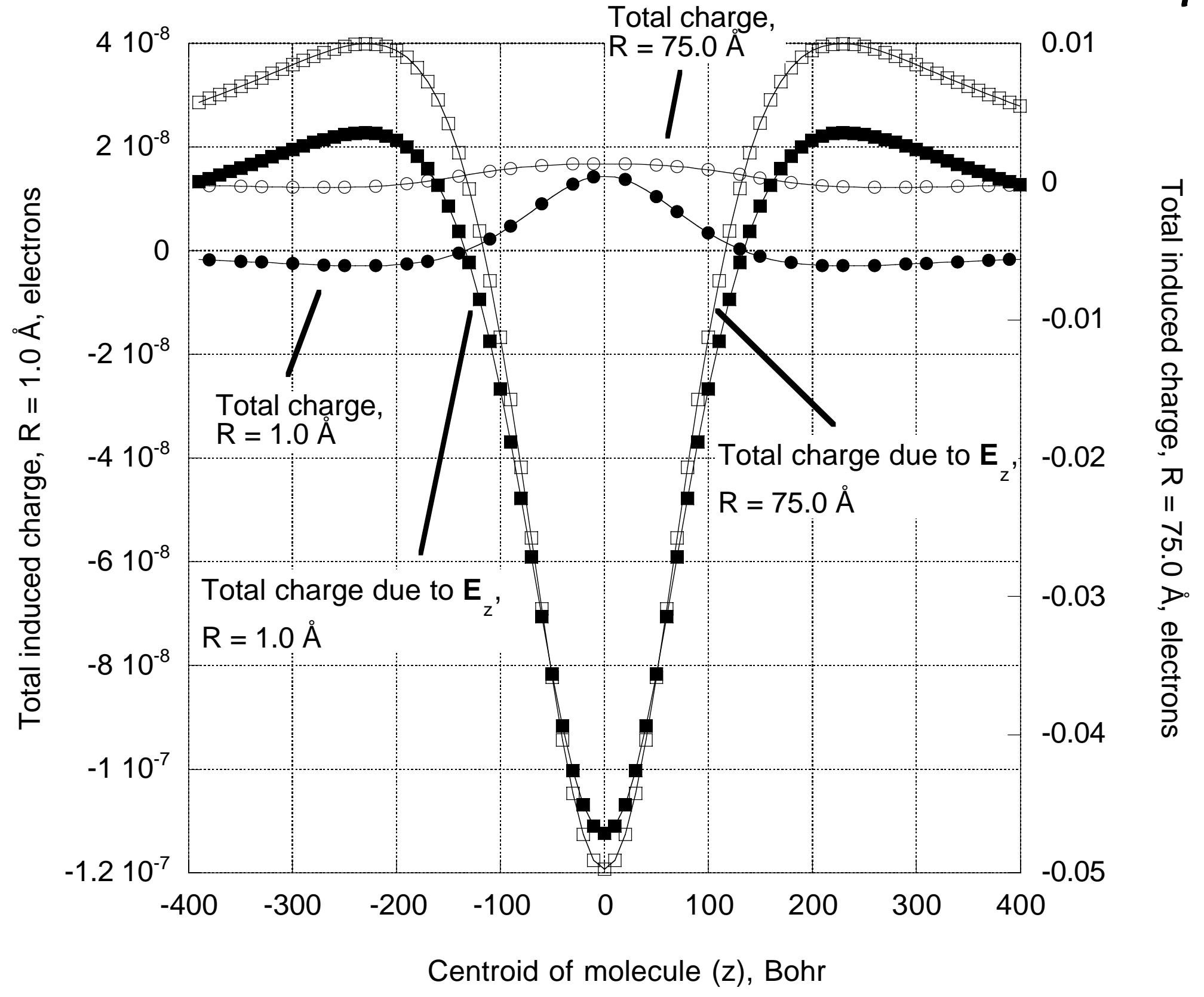

Fig. 5

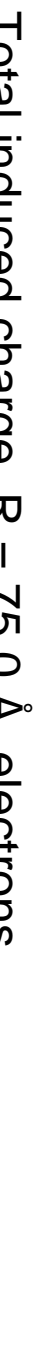


Fig. 6

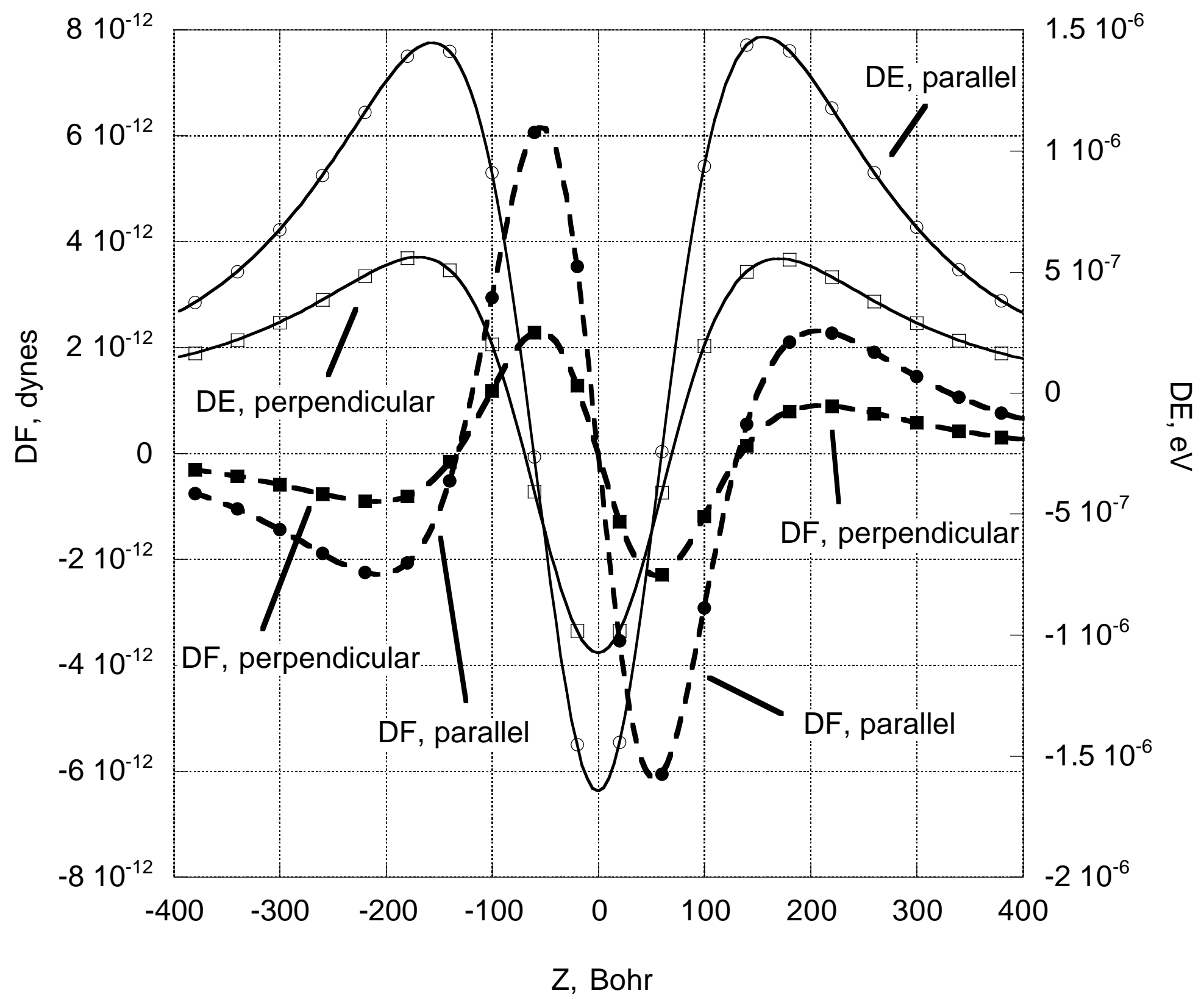



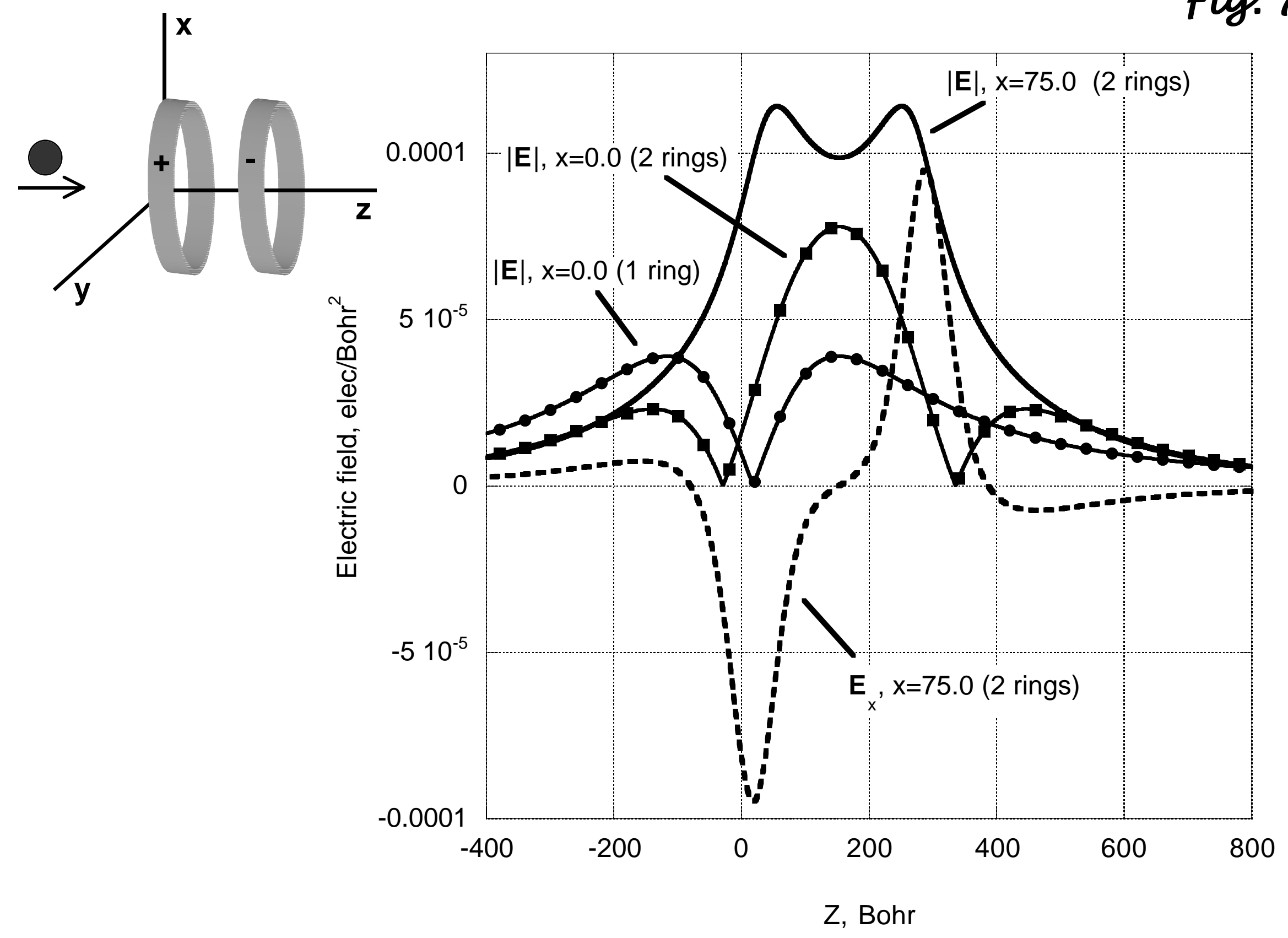
Fig. 8

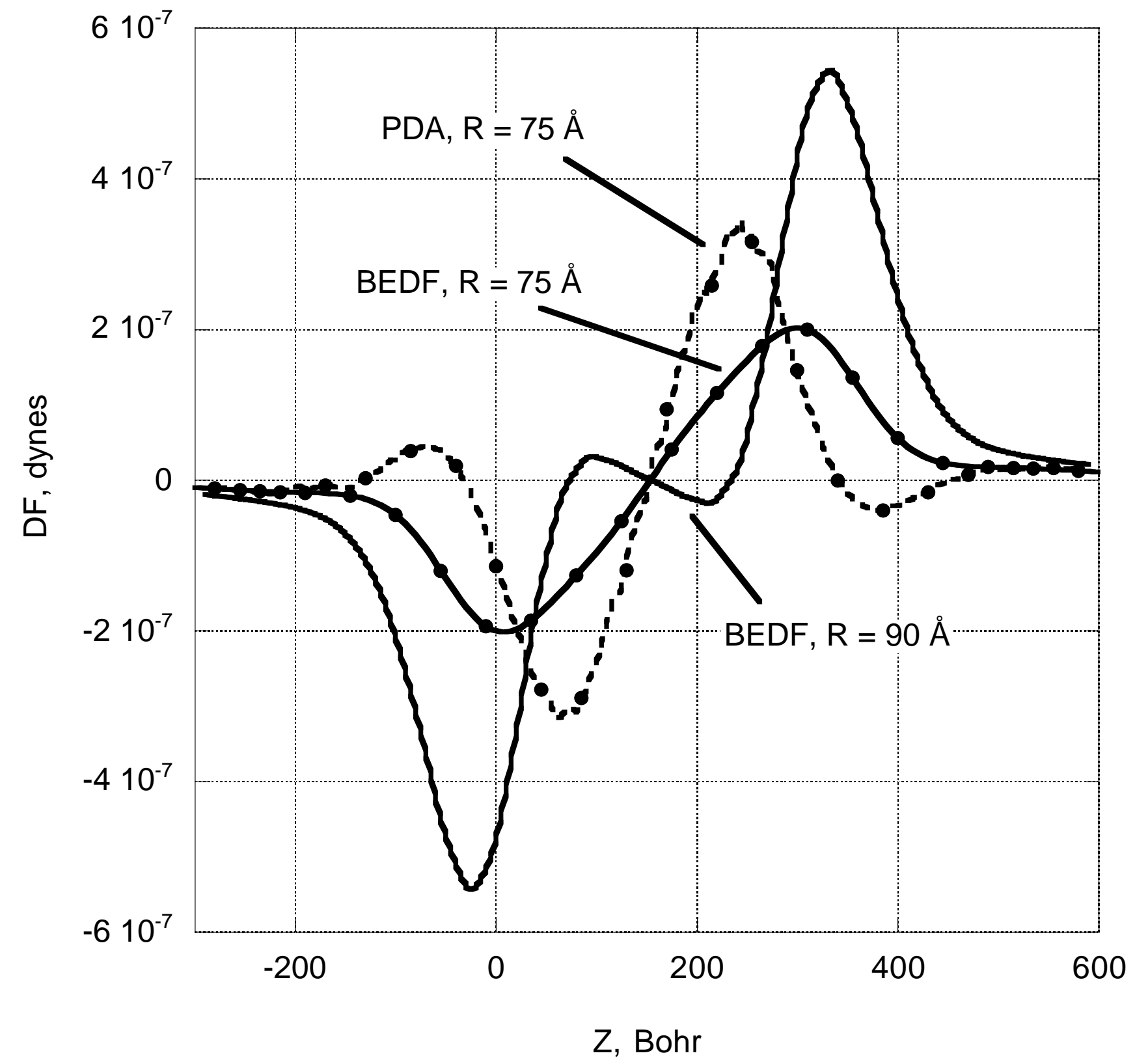

